\title{
Revisão - Efeito dos metais doadores nas propriedades elétricas e microestruturais dos varistores cerâmicos à base de $\mathrm{SnO}_{2}$
}

\section{(Review - Effect of donor metals on the electrical and microstructural properties of $\mathrm{SnO}_{2}$-based ceramic varistors)}

\author{
M. M. Oliveiral, J. H. G. Rangel ${ }^{1}$, V. C. de Sousa ${ }^{2}$, E. Longo ${ }^{3}$, R. N. R. Filho ${ }^{4}$ \\ ${ }^{I}$ CEFET-MA - DAQ, Av. Getúlio Vargas 04, S. Luís, MA 65025-001 \\ ${ }^{2}$ DEMAT, Universidade Federal do Rio Grande do Sul - UFRGS, Campus do Vale \\ Av. Bento Gonçalves, 9500, Setor 4, P. 74, $1^{\circ}$ andar, S. 213, Porto Alegre, RS \\ ${ }^{3}$ CMDMC, LIEC, Instituto de Química, UNESP, C.P. 355, Araraquara, SP 14801-907 \\ ${ }^{4}$ Centrais Elétricas do Norte do Brasil S/A - ELETRONORTE, Av. dos Jequitibás, S. Luís, MA 65040-460
}

\begin{abstract}
Resumo
O estudo da adição de dopantes trivalentes é uma das principais pesquisas na área de varistores. Vários autores têm buscado entender os efeitos destes dopantes nas propriedades elétricas e microestruturais destas cerâmicas eletrônicas. Tanto metais de transição quanto terras raras são adicionados em cerâmicas à base de $\mathrm{SnO}_{2}$ a fim de verificar o seu comportamento. O que se tem observado é que alguns destes óxidos tais como $\mathrm{Cr}_{2} \mathrm{O}_{3} \mathrm{e} \mathrm{La}_{2} \mathrm{O}_{3}$ melhoram significativamente as propriedades elétricas dos varistores, enquanto que outros como o $\mathrm{Bi}_{2} \mathrm{O}_{3}$ e $\mathrm{Er}_{2} \mathrm{O}_{3}$, por exemplo, não produzem tal efeito. A evolução do desempenho do comportamento varistor tem sido também atribuída às espécies de oxigênio produzidas pela reação com estes dopantes. Esta revisão apresenta resultados de estudos recentes do comportamento varistor frente a adição de metais doadores .

Palavras-chave: varistores, propriedades elétricas, microestrutura.
\end{abstract}

\begin{abstract}
The study of trivalent donor dopants is one of the main research subjects in the varistor area. Several researchers have been studying these dopants aiming at a better understanding of the effects of their addition on the electrical and microstructural properties of these electronic ceramics. Both transition and rare earth metals have been added to $\mathrm{SnO}_{2}$-based ceramics with the purpose of assessing their behavior. It has been observed that some of these oxides, such as $\mathrm{Cr}_{2} \mathrm{O}_{3}$ and $\mathrm{La}_{2} \mathrm{O}_{3}$, improve significantly the electrical properties of the varistors, whereas other oxides, such as $\mathrm{Bi}_{2} \mathrm{O}_{3}$ and $\mathrm{Er}_{2} \mathrm{O}_{3}$, for example, do not produce such effect. Improvements in the varistor behavior have been attributed to the different oxygen species produced by the reactions with these dopants. This review shows what has been studied recently, in terms of the dopants incorporated into the $\mathrm{SnO}_{2}$-based varistor lattices.
\end{abstract}

Keywords: varistors, electrical properties, microstructure.

\section{O EFEITO DOS DOPANTES TRIVALENTES}

A influência de óxidos trivalentes nas propriedades dos varistores vem sendo estudada por vários autores $[1-4,7$, $10,12,13,22]$. Estes óxidos não são apenas incorporados na composição química dos varistores para diminuir a resistividade elétrica dos grãos melhorando a não linearidade, mas também para aumentar a energia da barreira de voltagem devido a segregação do metal na junção grão-grão.

O comportamento do $\mathrm{Sb}_{2} \mathrm{O}_{3}$ em substituição ao $\mathrm{Nb}_{2} \mathrm{O}_{5} \mathrm{em}$ sistemas à base de $\mathrm{SnO}_{2}$ foi estudado [3]. Quando o óxido de estanho foi dopado com $\mathrm{Sb}_{2} \mathrm{O}_{3}$ e $\mathrm{CoO}$ foi verificado um alto coeficiente não linear $(\alpha=30)$ e um campo elétrico de ruptura de $120 \mathrm{~V} \cdot \mathrm{mm}^{-1}$. A adição do $\mathrm{CoO}$ levou à criação de vacâncias de oxigênio que, além de contribuir com o processo de sinterização do $\mathrm{SnO}_{2}$, também contribuiu com a formação da barreira de voltagem grão-grão. O óxido de antimônio agiu como doador, aumentando a condutividade do óxido de estanho. Em pequenas concentrações, como pentavalente, o antimônio está presente de acordo com a reação abaixo:

$$
\mathrm{Sb}_{2} \mathrm{O}_{3}+\mathrm{SnO}_{2} \stackrel{\mathrm{O}_{2}}{\longrightarrow} 2 \mathrm{Sb}_{\mathrm{Sn}}^{\cdot}+2 \mathrm{e}^{\prime}+4 \mathrm{O}_{\mathrm{O}}^{\mathrm{x}}+1 / 2 \mathrm{O}_{2}
$$

Por meio dessa equação pode-se observar que numa atmosfera rica em $\mathrm{O}_{2}$ háa formação de defeitos substitucionais e elétrons livres, que pode melhorar as características elétricas destas cerâmicas.

Outro dopante trivalente amplamente avaliado para promover melhores características a varistores $\mathrm{SnO}_{2}$ é o $\mathrm{Bi}_{2} \mathrm{O}_{3}$, que quando adicionado ao sistema $(98,95-\mathrm{x}) \mathrm{mol} \%$ 
$\mathrm{SnO}_{2}+1,00 \mathrm{~mol} \% \mathrm{CoO}+0,05 \mathrm{~mol} \% \mathrm{Nb}_{2} \mathrm{O}_{5}+\mathrm{x} \mathrm{mol} \% \mathrm{Bi}_{2} \mathrm{O}_{3}$, $\operatorname{com} \mathrm{x}=0 / 0,05 / 0,10 / 0,30 / 0,50$, obteve-se uma variação em $\alpha$ entre 8 e 10 e $\mathrm{E}_{\mathrm{r}}$ entre 880 e 1178 V.cm ${ }^{-1}$. Outra observação importante é que o Bi promoveu o crescimento do tamanho médio de grão e não formou fase líquida no processo de sinterização [4].

Ao adicionar $\mathrm{Co}_{2} \mathrm{O}_{3}$ em varistores à base de $\mathrm{SnO}_{2}$ dopados com $\mathrm{Ta}_{2} \mathrm{O}_{5}$, foram observadas significantes variações nas propriedades microestruturais e elétricas deste material [5]. Para amostra dopada com $0,1 \mathrm{~mol} \% \mathrm{Co}_{2} \mathrm{O}_{3}$, foi encontrado um coeficiente de não linearidade igual a 33 e um alto campo elétrico de ruptura $\left(\mathrm{E}_{\mathrm{r}}=872 \mathrm{~V} \cdot \mathrm{mm}^{-1}\right)$, além de uma baixa constante dielétrica relativa $\varepsilon_{\mathrm{r}}=598$ (medida a $1 \mathrm{kHz}$ ). Contudo, esta percentagem de dopante não foi suficiente para densificação da cerâmica à base de $\mathrm{SnO}_{2}$. A densidade relativa desta amostra foi $85,8 \%$ menor que nas dopadas com $0,3 / 0,5 / 1,2 \mathrm{~mol} \%$, que foram em torno de $98 \%$. O alto campo elétrico de ruptura e a baixa constante dielétrica relativa para as amostras dopadas com $0,1 \mathrm{~mol} \% \mathrm{Co}_{2} \mathrm{O}_{3}$ foram principalmente atribuídos à baixa densificação e ao pequeno tamanho médio de grão. As medidas de altura da barreira de potencial, $\phi_{\mathrm{b}}$, e resistência de contorno de grão, $\mathrm{R}_{\mathrm{GB}}$, indicaram que $\mathrm{Co}_{\mathrm{Sn}}^{\mathrm{x}}$ deve estar localizado na camada de depleção, sendo importante para formação da barreira de potencial no contorno de grão.

$\mathrm{O}$ varistor $98,85 \% \mathrm{SnO}_{2} .1,0 \% \mathrm{CoO} .0,05 \% \mathrm{Ta}_{2} \mathrm{O}_{5} .0,10 \%$ $\mathrm{Cr}_{2} \mathrm{O}_{3}$ sinterizado a $1350^{\circ} \mathrm{C}$ por $2 \mathrm{~h}$ com taxa de resfriamento de 2,10 e $15{ }^{\circ} \mathrm{C} \mathrm{min}^{-1}$ foi analisado [6]. Foi verificado o comportamento varistor somente quando foram usadas taxas de $2{ }^{\circ} \mathrm{C} \cdot \mathrm{min}^{-1}\left(\alpha=2\right.$ e $\left.\mathrm{E}_{\mathrm{r}}=4670 \mathrm{~V} \mathrm{~cm}{ }^{-1}\right)$ e $10{ }^{\circ} \mathrm{C} \cdot \mathrm{min}^{-1}$ $\left(\alpha=24\right.$ e $\left._{\mathrm{r}}=5530 \mathrm{~V} \mathrm{~cm}^{-1}\right)$.

Para avaliar a substituição do $\mathrm{Nb}$ por $\mathrm{Ta}$, foram estudadas as características elétricas do sistema $\mathrm{SnO}_{2} . \mathrm{CoO}$. $\mathrm{Ta}_{2} \mathrm{O}_{5}$ dopado com $0,05 \mathrm{~mol} \%$ de $\mathrm{Cr}_{2} \mathrm{O}_{3}$ e $\mathrm{Al}_{2} \mathrm{O}_{3}$ [7]. Foram determinados $\alpha=28$ e $\quad \mathrm{E}_{\mathrm{r}}=2400 \mathrm{~V} \cdot \mathrm{cm}^{-1}$ para o sistema dopado com $\mathrm{Cr}_{2} \mathrm{O}_{3}$ e $\alpha=12$ e E $=2016$ V.cm-1 para o sistema dopado com $\mathrm{Al}_{2} \mathrm{O}_{3}$.

Posteriormente, ao estudar o sistema $98,90 \% \mathrm{SnO}_{2} .1,00 \%$ CoO.0,05\% $\quad \mathrm{Nb}_{2} \mathrm{O}_{5} .0,05 \% \quad \mathrm{Cr}_{2} \mathrm{O}_{3}$, foi verificado que o valor da voltagem por barreira, $v_{b}$, variava com o tempo de sinterização [8]. Barreiras elétricas estáveis entre 2,0 e $2,4 \mathrm{~V} /$ barreira produziam varistores com coeficientes não lineares maiores que 35 , independentemente da temperatura de sinterização. A variação do tamanho médio de grão em relação ao tempo de sinterização foi de $45,1 \%$ para sinterização a $1300{ }^{\circ} \mathrm{C}$ e $33,6 \%$ para a sinterização a $1350{ }^{\circ} \mathrm{C}$, considerando um tempo mínimo de 1 h e um máximo de 12 h, com consideráveis variações nos respectivos campos elétricos de ruptura, mostrando assim que o tempo de sinterização é uma variável muito importante e que não pode ser desprezado no projeto de produção de varistores à base de $\mathrm{SnO}_{2}$.

Avaliando o efeito do $\mathrm{Cr}_{2} \mathrm{O}_{3}$, foi estudado o mesmo sistema variando a concentração em 0/0,05/0,06/0,065/0,07 mol\% $\mathrm{Cr}_{2} \mathrm{O}_{3}$ a uma temperatura de sinterização de $1350{ }^{\circ} \mathrm{C}$ e patamar para sinterização de 1 h [9]. Foi encontrado que o campo elétrico de ruptura aumentava de 400 a 1000 V.mm . $^{1}$ Foi observado que a permissividade elétrica relativa passa de 2022 a 147 com o aumento da concentração de $\mathrm{Cr}_{2} \mathrm{O}_{3}$ de 0 a $0,07 \mathrm{~mol} \%$. Para a concentração de $0,06 \mathrm{~mol} \% \mathrm{Cr}_{2} \mathrm{O}_{3}$ foi encontrado um coeficiente não linear 52. Estes resultados são extremamente interessantes, visto que em sistemas varistores dopados com $\mathrm{Cr}_{2} \mathrm{O}_{3}$ o excesso de oxigênio no contorno de grão pode ser controlado pela presença de átomos de cromo segregados ou precipitados como uma nova fase [10-13].

Foi também investigado como a adição de $\mathrm{Cr}_{2} \mathrm{O}_{3}$ afetava a heterogeneidade microestrutural e características não ohmicas de $\mathrm{SnO}_{2}\left(\mathrm{Co}_{\mathrm{x}} \mathrm{Mn}_{1-\mathrm{x}}\right) \mathrm{O}$ com $\mathrm{x}$ variando de 0 a $1 \mathrm{~mol} \%$ [14]. A presença de cromo promoveu um efeito significativo na característica não ohmica quando $\mathrm{x}=1$. Entretanto, essa característica diminuiu quando $\mathrm{x}$ variou de 1 a 0 . Tal comportamento foi explicado considerando o aumento das junções heterogêneas dentro do sistema, acompanhado pelo excesso de precipitados no ponto triplo na região de contorno de grão devido ao $\mathrm{MnO}$. A presença destes precipitados causou um aumento na corrente de fuga em resposta da criação de barreiras não efetivas. $\mathrm{O}$ efeito produzido pelo tratamento térmico deste sistema em atmosferas ricas em oxigênio e nitrogênio sugere que o $\mathrm{Cr}_{2} \mathrm{O}_{3}$ é mais susceptível ao oxigênio, que se concentra no contorno de grão melhorando as propriedades não ohmicas do sistema.

$\mathrm{O}$ efeito na microestrutura e propriedade elétrica do sistema $\mathrm{SnO}_{2}$ dopados com $\mathrm{CoO}$ e $\mathrm{Ta}_{2} \mathrm{O}_{5}$ com a adição de $\mathrm{Gd}_{2} \mathrm{O}_{3}$ foi estudado [15]. O campo elétrico de ruptura aumentou significativamente de $720 \mathrm{~V} \cdot \mathrm{mm}^{-1}$ para 1445 V.mm ${ }^{-1}$ e sua constante dielétrica relativa diminuiu de 833 a 330 para concentração de $\mathrm{Gd}_{2} \mathrm{O}_{3}$ superior a $1,2 \mathrm{~mol} \%$. $\mathrm{O}$ significativo decréscimo no tamanho médio de grão foi observado e atribuído à segregação de $\mathrm{Gd}_{2} \mathrm{O}_{3}$ no contorno de grão. Para concentração de $0,8 \mathrm{~mol} \% \mathrm{Gd}_{2} \mathrm{O}_{3}$ encontraram $\alpha$ 24,0 e $\mathrm{E}_{\mathrm{r}} 1125 \mathrm{~V} \cdot \mathrm{mm}^{-1}$ e para $1,2 \mathrm{~mol} \% 23,0$ e $1355 \mathrm{~V} \cdot \mathrm{mm}^{-1}$, respectivamente.

$\mathrm{O}$ efeito de $\mathrm{Dy}_{2} \mathrm{O}_{3}$ na microestrutura e nas propriedades elétricas de varistores à base de $\mathrm{SnO}_{2}$ também tem sido estudado [16]. As amostras com $1 \mathrm{~mol} \%$ de $\mathrm{Dy}_{2} \mathrm{O}_{3}$ no sistema (99,15-x)\% $\quad \mathrm{SnO}_{2} .0,75 \% \quad \mathrm{Co}_{2} \mathrm{O}_{3} \cdot 0,1 \% \quad \mathrm{Nb}_{2} \mathrm{O}_{5} \cdot \mathrm{x} \%$ $\mathrm{Dy}_{2} \mathrm{O}_{3}$ apresentaram a melhor não linearidade com alto valor de $\alpha(26,3)$. O tamanho médio de grão diminuiu de 16,7 para $8,3 \mu \mathrm{m}$ e o campo elétrico de ruptura aumentou de 177,9 para $742,2 \mathrm{~V} \cdot \mathrm{mm}^{-1}$.

$\mathrm{O}$ efeito de $\mathrm{Er}_{2} \mathrm{O}_{3}$ também foi investigado no sistema varistor $\mathrm{SnO}_{2} \cdot \mathrm{CoO} \cdot \mathrm{Ta}_{2} \mathrm{O}_{5}$ sinterizado a $1400{ }^{\circ} \mathrm{C}$ [17]. Foi reportado que todas as amostras apresentaram uma excelente característica não linear e a amostra com $0,5 \mathrm{~mol} \% \mathrm{Er}_{2} \mathrm{O}_{3}$ apresentou um alto coeficiente não linear $(43,7)$. Com o aumento da concentração do $\mathrm{Er}_{2} \mathrm{O}_{3}$ de 0,10 para $1,0 \mathrm{~mol} \%$ o tamanho médio de grão diminuiu de 21,2 para 10,6 um e o campo elétrico de ruptura aumentou de 208 para 459 $\mathrm{V} . \mathrm{mm}^{-1}$, enquanto que a permissividade elétrica relativa caiu de 2440 para 1210.

$\mathrm{O}$ efeito do $\mathrm{Er}_{2} \mathrm{O}_{3}$ no sistema $(98,25-\mathrm{x}) \mathrm{SnO}_{2}+$ $0,75 \% \mathrm{Co}_{2} \mathrm{O}_{3}+0,1 \% \mathrm{Nb}_{2} \mathrm{O}_{5}+x \% \mathrm{Er}_{2} \mathrm{O}_{3}$, com $x=0 / 0,1 / 0,5 /$ 
1,0/2,0 foi estudado [18]. Foi observado o mesmo efeito nas temperaturas de sinterização 1250,1300 e $1350{ }^{\circ} \mathrm{C}$. Para todas as amostras a densidade relativa foi maior que $98 \%$ e o coeficiente não linear medido ficou em torno de 16 . Foi observado que a adição de $\mathrm{Er}_{2} \mathrm{O}_{3}$ promoveu um grande efeito nas propriedades elétricas do sistema, aumentando substancialmente o campo elétrico de ruptura e baixando significativamente a resistividade.

Os sistemas varistores $\mathrm{SnO}_{2} \cdot \mathrm{Co}_{3} \mathrm{O}_{4} \cdot \mathrm{Nb}_{2} \mathrm{O}_{5} \cdot \mathrm{Fe}_{2} \mathrm{O}_{3}$ (SCNF) e $\mathrm{SnO}_{2} \cdot \mathrm{ZnO} \cdot \mathrm{Nb}_{2} \mathrm{O}_{5} \cdot \mathrm{Fe}_{2} \mathrm{O}_{3}$ (SZNF) foram estudados por meio de microscopia eletrônica de varredura e de transmissão a fim de verificar a possibilidade de existência de uma segunda fase nas regiões de grão e de contorno de grão [19]. Foi observada a formação da fase $\mathrm{Co}_{1,5} \mathrm{FeSn}_{0,5} \mathrm{O}_{4}$ para o sistema $\mathrm{SCNF}$ e $\mathrm{Zn}_{2} \mathrm{SnO}_{4}$ para o $\mathrm{SZNF}$, associando-as à melhora da não linearidade dos sistemas varistores. Para o sistema contendo $\mathrm{Y}_{2} \mathrm{O}_{3}$, além da fase pirocloro, $\mathrm{Y}_{2} \mathrm{Sn}_{2} \mathrm{O}_{7}$, também foram observadas partículas de $\mathrm{Co}_{2} \mathrm{SnO}_{4}$, fases estas que também promovem significativamente as propriedades elétricas do varistor [20].

Foi adicionado $\mathrm{La}_{2} \mathrm{O}_{3}$ em quantidades de 0,05 a 0,075 mol\% ao sistema $(98,95-\mathrm{x}) \% \quad \mathrm{SnO}_{2} .1,0 \% \quad \mathrm{CoO} .0,05 \%$ $\mathrm{Nb}_{2} \mathrm{O}_{5} \times \mathrm{La}_{2} \mathrm{O}_{3}$ [21]. As amostras foram sinterizadas por $2 \mathrm{~h}$ a $1300{ }^{\circ} \mathrm{C}$. A adição de $\mathrm{La}_{2} \mathrm{O}_{3}$ inibiu o crescimento de grão e induziu um aumento na porosidade intergranular para concentrações maiores que $0,05 \mathrm{~mol} \%$. As amostras dopadas com $0,05 \mathrm{~mol} \% \mathrm{La}_{2} \mathrm{O}_{3}$ apresentaram $\alpha=16,8$ e $\mathrm{E}_{\mathrm{r}}$ $=3369{\mathrm{~V} . \mathrm{cm}^{-1}}^{\mathrm{e}}$ as amostras dopadas com $0,075 \mathrm{~mol} \%$ de $\mathrm{La}_{2} \mathrm{O}_{3} \alpha=12,7$ e 6536 V.cm ${ }^{-1}$. Portanto, houve um aumento

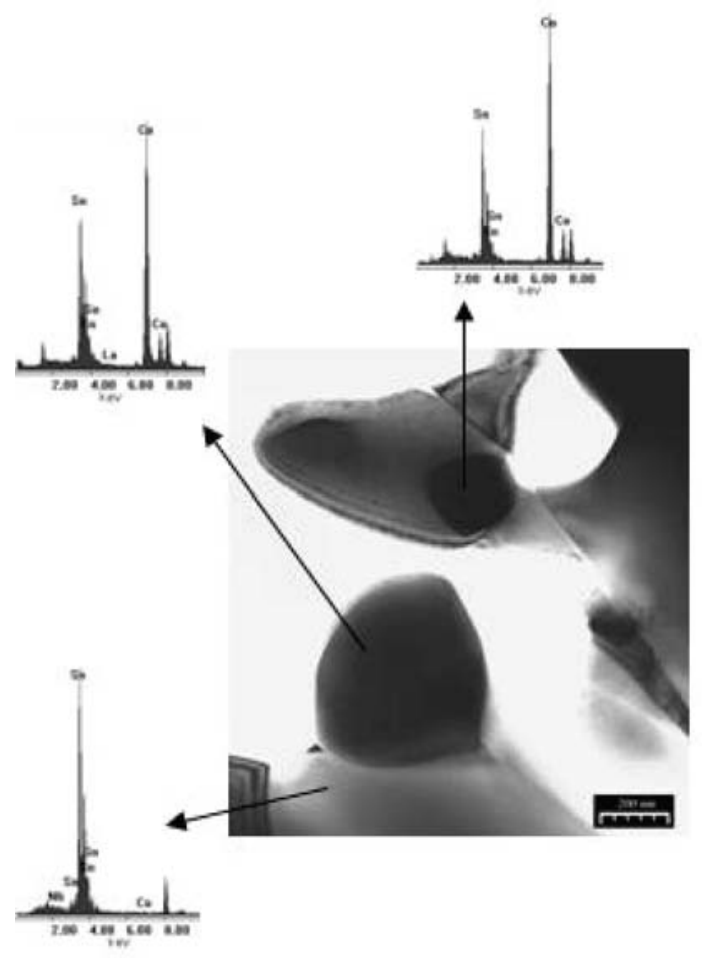

do coeficiente de não linearidade e campo elétrico de ruptura ao sistema ternário usado como base cujo $\alpha$ era igual a 8 e $\mathrm{E}_{\mathrm{r}}$ igual a $1870 \mathrm{~V} \cdot \mathrm{cm}^{-1}$.

Foi também investigada a presença de uma segunda fase em sistemas varistores de composição molar $98,665 \% \mathrm{SnO}_{2}$ $+1,00 \% \mathrm{CoO}+0,035 \% \mathrm{Nb}_{2} \mathrm{O}_{5}+0,30 \% \mathrm{X}, \mathrm{X}=\mathrm{La}_{2} \mathrm{O}_{3}$ ou $\mathrm{Pr}_{2} \mathrm{O}_{3}$ (SCNLa ou SCNPr) [22]. Para estes sistemas, foram encontradas as fases $\mathrm{Co}_{2} \mathrm{SnO}_{4}$ e $\mathrm{Pr}_{2} \mathrm{Sn}_{2} \mathrm{O}_{7}$ por meio da técnica de espectrometria de energia dispersiva, indicando principalmente que tais precipitados se encontravam nas junções triplas da microestrutura. Não foi encontrada uma fase secundária tal como $\mathrm{La}_{2} \mathrm{Sn}_{2} \mathrm{O}_{7}$, possivelmente devido à formação de uma solução sólida de $\mathrm{La}_{2} \mathrm{O}_{3} \operatorname{com~} \mathrm{SnO}_{2}$. Outros autores têm estudado o comportamento de $\mathrm{La}_{2} \mathrm{O}_{3}$ e observado que a adição deste dopante inibe o crescimento de grão e melhora as propriedades não lineares dos varistores [23,24]. A micrografia por MET pode ser observada na Fig. 1.

$\mathrm{O}$ efeito de $\mathrm{In}_{2} \mathrm{O}_{3}$ também tem sido investigado [24, 25]. Em sistemas à base de $\mathrm{SnO}_{2}$ contendo $\mathrm{Co}_{2} \mathrm{O}_{3}$ e $\mathrm{Nb}_{2} \mathrm{O}_{5}$ sinterizados a $1350{ }^{\circ} \mathrm{C}$, no qual um $\alpha$ máximo de 20,4 foi encontrado para concentração de $0,05 \mathrm{~mol} \%$ de $\operatorname{In}_{2} \mathrm{O}_{3}$ e o tamanho médio de grão tem diminuído com a adição de maiores concentrações, assim como a permissividade dielétrica relativa. Já quando adiciona a varistores contendo $\mathrm{Ta}_{2} \mathrm{O}_{3}$, em substituição ao $\mathrm{Nb}_{2} \mathrm{O}_{5}$, obtém-se coeficiente não linear em torno de 51. Porém, o comportamento da permissividade relativa e tamanho médio de grão são os mesmos.

A dopagem com $\operatorname{Pr}_{2} \mathrm{O}_{3}$ também tem sido estudada em a)

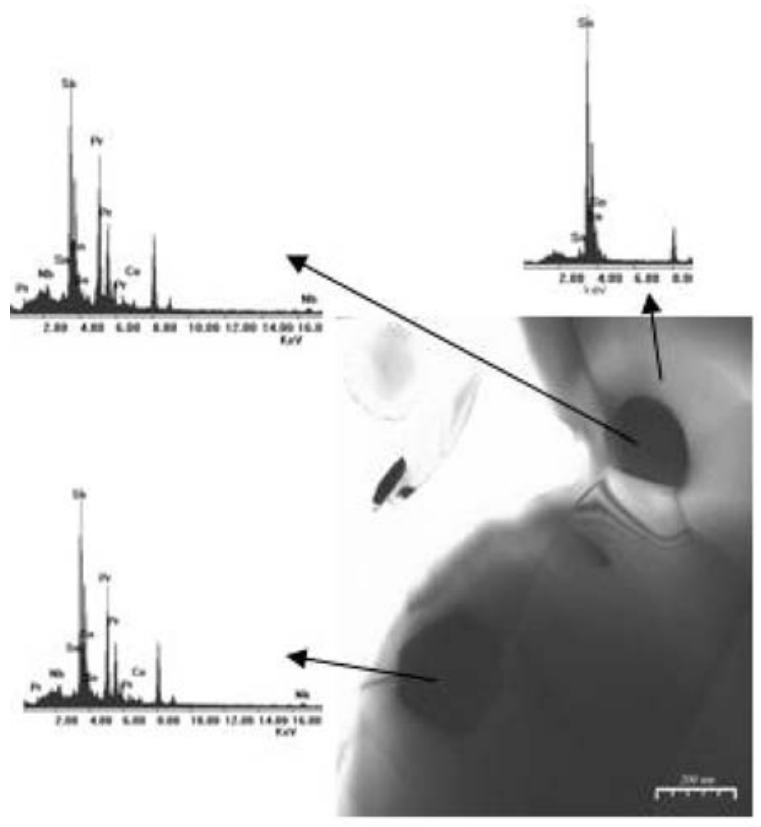

Figura 1: Micrografias obtidas por MET para: (a) $\mathrm{SCN}-0,30 \% \mathrm{La}_{2} \mathrm{O}_{3}$ e (b) $\mathrm{SCN}-0,30 \% \operatorname{Pr}_{2} \mathrm{O}_{3}$ com os padrões de raios X [22]. [Figure 1: TEM micrographs of: (a) SCN-0.30\% $\mathrm{La}_{2} \mathrm{O}_{3}$ and (b) $\mathrm{SCN}^{2}-0.30 \% \mathrm{Pr}_{2} \mathrm{O}_{3}$ with the X-ray patterns [22].] 
Tabela I - Temperatura de sinterização, tempo de sinterização, tamanho médio de grão e parâmetros elétricos de alguns varistores a base de $\mathrm{SnO}_{2}$.

[Table I - Sintering temperature, sintering time, average grain size and electrical parameteres of some $\mathrm{SnO}_{2}$-based varistors.]

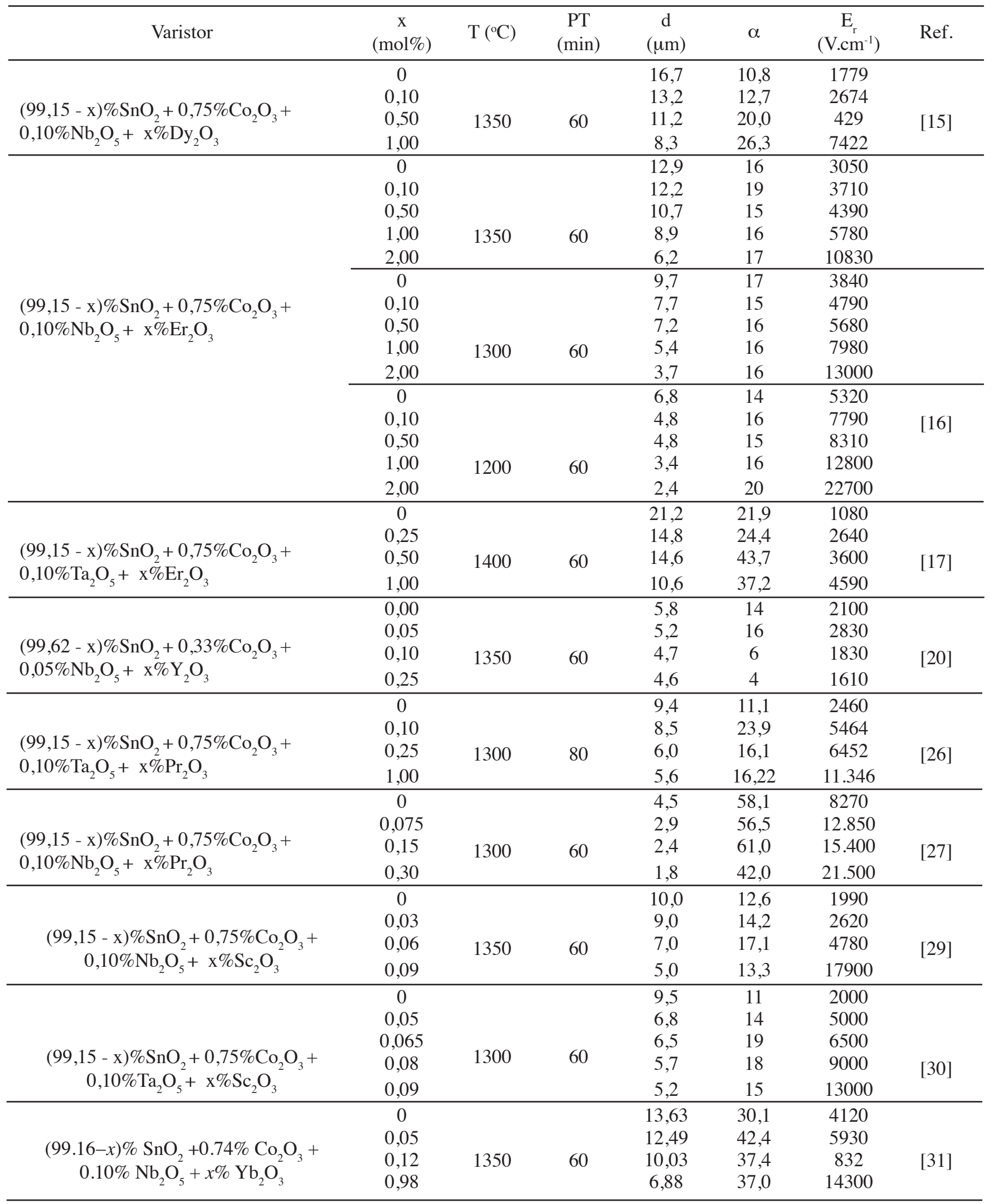


sistemas varistores a base de $\mathrm{SnO}_{2}$ dopados com $\mathrm{Nb}$ e $\mathrm{Co}(\mathrm{III})$ com altos coeficientes não lineares foram encontrados [26, 27]. Para concentração de $0,15 \mathrm{~mol} \%$ de $\operatorname{Pr}_{2} \mathrm{O}_{3}$ o valor de $\alpha$ encontrado foi 61 . Sem $\operatorname{Pr}_{2} \mathrm{O}_{3}$ o coeficiente de não linearidade ficou em torno de 58,1. Os comportamentos microestrutural, elétrico e dielétrico também são influenciados à medida que se varia a concentração deste dopante. Em sistemas dopados com Ta e Co(III) tal comportamento é observado, porém o valor de $\alpha$ é bem menor, 23,9 para concentração de 0,10 mol\%. Podem-se observar que para os sistemas contendo $\mathrm{Ta}_{2} \mathrm{O}_{5}$ o tamanho médio de grão é quase três vezes maiores que os contendo $\mathrm{Nb}_{2} \mathrm{O}_{5}$, o que pode estar influenciando nas propriedades não lineares destas cerâmicas. A Tabela I mostra estes valores.

Já para sistemas $\mathrm{SnO}_{2} \cdot \mathrm{Co}_{2} \mathrm{O}_{3} \cdot \mathrm{Nb}_{2} \mathrm{O}_{5}$ dopados com $\mathrm{Sc}_{2} \mathrm{O}_{3}$ o valor máximo de $\alpha$ foi 17,1 para concentração de 0,06 mol\% do dopante, valor bem menor que os observados para os sistemas contendo $\operatorname{Pr}_{2} \mathrm{O}_{3}$. No entanto, apesar do decréscimo da não linearidade, o sistema mantem os demais comportamentos como o aumento do campo elétrico de ruptura e a diminuição do tamanho médio de grão [28]. Neste sistema a temperatura de sinterização foi $1350{ }^{\circ} \mathrm{C}$ enquanto que para o sistema contendo $\operatorname{Pr}_{2} \mathrm{O}_{3}$ foi $1300{ }^{\circ} \mathrm{C}$. Tal dopante também tem sido estudado com sistemas $\mathrm{SnO}_{2} \cdot \mathrm{Co}_{2} \mathrm{O}_{3} \cdot \mathrm{Ta}_{2} \mathrm{O}_{5}$ e $\mathrm{SnO}_{2} \cdot \mathrm{Co}_{2} \mathrm{O}_{3} \cdot \mathrm{Nb}_{2} \mathrm{O}_{5} \cdot \mathrm{Cr}_{2} \mathrm{O}_{3}[29,30]$.

$\mathrm{O} \mathrm{Yb}_{2} \mathrm{O}_{3}$ quando adicionado ao sistema $(99,16-x) \mathrm{mol} \%$ $\mathrm{SnO}_{2}+0,74 \mathrm{~mol} \% \mathrm{Co}_{2} \mathrm{O}_{3}+0,10 \mathrm{~mol} \% \mathrm{Nb}_{2} \mathrm{O}_{5}+x \mathrm{~mol} \%$ $\mathrm{Yb}_{2} \mathrm{O}_{3}$, com $x=0 / 0,05 / 0,20 / 0,98$, promove melhorias significativas nas propriedades elétricas, atingindo valor de $\alpha 42,4$ para $0,98 \mathrm{~mol} \%$ de $\mathrm{Yb}_{2} \mathrm{O}_{3}$ [31]. Os valores de alguns sistemas citados são mostrados na Tabela I. Esses dopantes influenciam no tamanho médio de grão e, conseqüentemente, as propriedades elétricas.

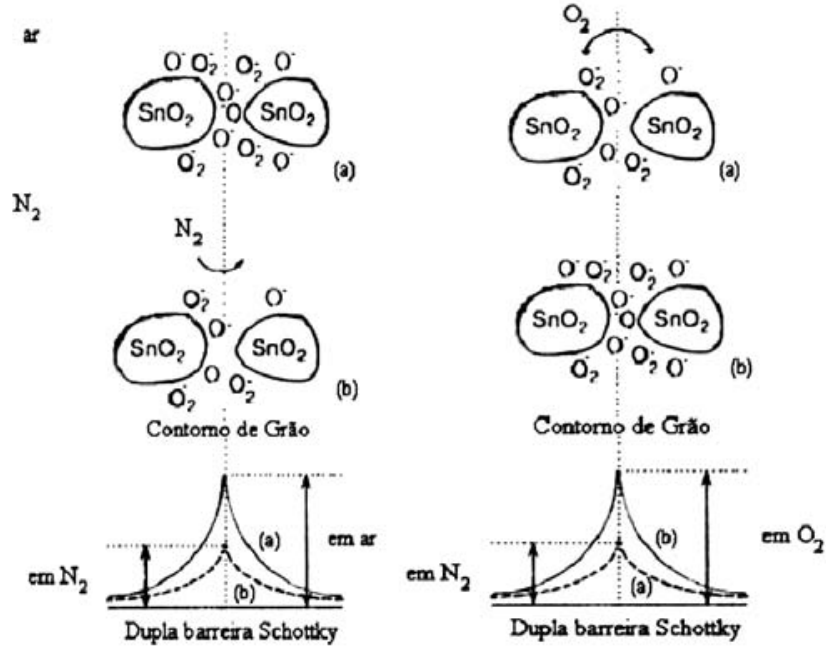

Figura 2: Desenho esquemático da relação entre a superfície coberta de oxigênios adsorvidos em varistores a base de $\mathrm{SnO}_{2}$ e a altura da dupla barreira Schottky no contorno de grão [33].

[Figure 2: Schematic drawing of oxygen recovered surface in $\mathrm{SnO}_{2}$-based varistors and double Schottky barrier at the grain boundary [33].]
Tabela II - Campo elétrico de ruptura e número de barreiras efetivas para os sistemas estudados [33].

[Table II - Breakdown electric field and number of effective voltage barriers of the $\mathrm{SnO}_{2}$-based varistors [33].]

\begin{tabular}{ccccccc}
\hline Varistor & \multicolumn{3}{c}{$\mathrm{E}_{\mathrm{r}}\left({\mathrm{V} . \mathrm{cm}^{-1}}^{-1}\right.$} & \multicolumn{3}{c}{$v_{\mathrm{b}}$ (V/barreira) } \\
\hline SCNLa & ar & $N_{2}$ & $\mathrm{O}_{2}$ & ar & $N_{2}$ & $O_{2}$ \\
0,05 & 7354 & 457 & 7172 & 1,8 & 0,1 & 1,8 \\
0,10 & 6697 & 1277 & 6158 & 1,5 & 0,3 & 1,4 \\
0,15 & 5022 & 2751 & 7959 & 1,0 & 0,5 & 1,6 \\
0,30 & 11083 & 3009 & 9636 & 1,8 & 0,5 & 1,5 \\
& & & & & & \\
SCNAl & & & & & & \\
0,05 & 8292 & 6637 & 7594 & 3,4 & 2,7 & 3,1 \\
0,10 & 8804 & 7282 & 7968 & 3,3 & 2,7 & 2,9 \\
0,15 & 10835 & 9267 & 11385 & 2,5 & 2,1 & 2,6 \\
0,30 & 9229 & 9368 & 10372 & 2,0 & 2,1 & 2,3 \\
& & & & & & \\
SCNPr & & & & & & \\
0,05 & 6908 & 5725 & 7129 & 2,2 & 1,8 & 2,3 \\
0,10 & 5544 & 4929 & 5058 & 1,8 & 1,6 & 1,6 \\
0,15 & 7249 & 2899 & 8129 & 2,2 & 0,9 & 2,4 \\
0,30 & 7363 & 2164 & 11138 & 2,0 & 0,6 & 3,0 \\
& & & & & & \\
SCNCe & & & & & & \\
0,05 & 4288 & 2574 & 4302 & 1,5 & 0,9 & 1,5 \\
0,10 & 3844 & 3095 & 3446 & 1,9 & 1,5 & 1,7 \\
0,15 & 3243 & 2617 & 2787 & 1,8 & 1,4 & 1,5 \\
0,30 & 4403 & 5367 & 5164 & 1,4 & 1,7 & 1,6 \\
SCNCrLa & 11849 & 9495 & 11571 & 2,3 & 1,8 & 2,2 \\
SCNCrAl & - & - & - & - & - & - \\
SCNCrPr & 6866 & 5877 & 5969 & 2,7 & 2,3 & 2,3 \\
SCNCrCe & 5975 & 5580 & 5598 & 2,1 & 2,0 & 2,0 \\
\hline
\end{tabular}

(98,965-y)\% $\mathrm{SnO}_{2} .0,035 \% \mathrm{Nb}_{2} \mathrm{O}_{5} .1,00 \%$ CoO. $\mathrm{yX}_{2} \mathrm{O}_{3}(\mathrm{SCNX})$ (98,965-y)\% $\mathrm{SnO}_{2} \cdot 0,035 \% \mathrm{Nb}_{2} \mathrm{O}_{5} \cdot 1,00 \%$ CoO.yCeO $($ (SCNCe) $98,465 \% \mathrm{SnO}_{2} .0,035 \% \mathrm{Nb}_{2} \mathrm{O}_{5} .1,00 \% \mathrm{CoO} .0,25 \% \mathrm{Cr}_{2} \mathrm{O}_{3} 0,25 \% \mathrm{X}_{2} \mathrm{O}_{3}$ (SCNCrX) $98,465 \% \mathrm{SnO}_{2} \cdot 0,035 \% \mathrm{Nb}_{2} \mathrm{O}_{5} \cdot 1,00 \% \mathrm{CoO} .0,25 \% \mathrm{Cr}_{2} \mathrm{O}_{3} 0,25 \% \mathrm{CeO}_{2}$ (SCNCrCe) Em que $\mathrm{X}=\mathrm{La}, \mathrm{Al}$, Pr e y = 0,05; 0,10; 0,15 e 0,30.

A adição de tais dopantes pode promover a incorporação de defeitos doadores na barreira de potencial, melhorando as propriedades elétricas. A equação geral na notação de Kröger-Vink para a incorporação destes óxidos é:

$$
\mathrm{M}_{2} \mathrm{O}_{3} \stackrel{\mathrm{SnO}_{2}}{\longrightarrow} 2 \mathrm{M}_{\mathrm{Sn}}^{\prime}+\mathrm{V}_{\mathrm{O}}^{*}+3 \mathrm{O}_{\mathrm{O}}^{\mathrm{x}}
$$

Ou seja, defeitos negativos estão se formando no contorno de grão e vacâncias de oxigênio estão sendo formadas na cerâmica a fim de melhorar a difusão do material [32].

Estes defeitos também podem ser responsáveis pela adsorção de oxigênio, que estão associados à formação da barreira do tipo Schottky na interface de acordo com as seguintes equações [13, 32-37]:

$$
\mathrm{O}_{2} \rightarrow \mathrm{O}_{2(\text { ad) }}^{\mathrm{x}}
$$




$$
\begin{aligned}
& \mathrm{M}_{\mathrm{Sn}}^{\prime}+\mathrm{O}_{2(\mathrm{ad})}^{\mathrm{x}} \rightarrow \mathrm{M}_{\mathrm{Sn}}^{\mathrm{x}}+\mathrm{O}_{2(\mathrm{ad})}^{\prime} \\
& \mathrm{M}_{\mathrm{Sn}}^{\prime}+\mathrm{O}_{2(\mathrm{ad})}^{\prime} \rightarrow \mathrm{M}_{\mathrm{Sn}}^{\mathrm{x}}+2 \mathrm{O}_{(\mathrm{ad})}^{\prime}
\end{aligned}
$$

contribuindo ainda mais nas propriedades elétricas dos sistemas varistores cerâmicos.

A mudança no campo elétrico de ruptura para um campo elétrico mais baixo sugere o decréscimo na concentração de defeitos aceitadores. Esta mudança é considerada na barreira Schottky, que é bem aceita como origem da não linearidade nos varistores [38-41], devido ao decréscimo na altura da barreira resultando num menor campo elétrico de ruptura, maior corrente de fuga e menor coeficiente de não linearidade [42]. Vários modelos explicam a formação da barreira por oxigênios adsorvidos em grãos de $\mathrm{ZnO}$ próximos ao contorno de grão e excesso de íons oxigênio no contorno de grão; estas espécies podem ser consumidas pelo gás hidrogênio especialmente a temperaturas elevadas [41-45].

O decréscimo em ambas as superfícies cobertas de oxigênio adsorvido e a concentração de excesso de íons oxigênio induzido pela reação do gás nitrogênio e, portanto, o decréscimo na altura da barreira, são responsáveis pelas pequenas mudanças no campo elétrico de ruptura na presença do gás nitrogênio como ilustrado na Fig. 2. A quantidade de oxigênio adsorvido por unidade de volume do $\mathrm{SnO}_{2}$ aumenta com a diminuição do tamanho de grão que leva a altos valores de campo elétrico de ruptura e conseqüentemente o número de contornos aumenta contribuindo para o melhoramento da sensibilidade. A hipótese de que um dos principais transportadores de carga ser essencialmente espécies de oxigênio adsorvidas no contorno de grão pode ser verificada por meio do tratamento térmico do sistema varistor à base de $\mathrm{SnO}_{2}$, previamente sinterizados em ar, em atmosferas de oxigênio e nitrogênio [13, 33]. A Tabela II mostra a variação da voltagem por barreira $\left(v_{b}\right)$ com diferentes atmosferas para alguns sistemas. Pode-se observar que o aumento e o decréscimo do campo elétrico de ruptura com diferentes atmosferas estão associados com o número de barreiras de voltagem efetivas. Na maioria dos sistemas os valores obtidos, ainda são pequenos comparados com a barreira de voltagem esperada para o $\mathrm{SnO}_{2}(\sim 3,5 \mathrm{~V})$. Sendo assim, o sistema mais próximo da voltagem esperada é o SCNAl, em que $97 \%$ das barreiras são efetivas. Os resultados da Tabela II sugerem fortemente que o oxigênio adsorvido na interface do contorno de grão é a principal fonte de cargas negativas minoritárias responsáveis pelo comportamento não linear do varistor à base de $\mathrm{SnO}_{2}$.

Esta revisão teve como finalidade mostrar o que se tem estudado nos últimos anos referentes a dopantes trivalentes em sistemas varistores à base de $\mathrm{SnO}_{2}$. Tais dopantes têm sido citados como os principais responsáveis pelo mecanismo de adsorção de oxigênio, responsáveis pelas propriedades elétricas destas cerâmicas varistoras, e vem sendo bem aceito pela comunidade científica. Dopantes de carga 3+ têm-se mostrado parte fundamental para a promoção e estabilidade das propriedades não lineares mais adequadas para a aplicação como varistores de alta tensão. $\mathrm{O}$ conhecimento da atuação desses dopantes na promoção das propriedades varistoras é de fundamental importância para o controle da faixa de atuação dos mesmos, daí a necessidade cada vez maior de se estudar, conhecer e dominar os mecanismos que governam tais propriedades.

\section{AGRADECIMENTOS}

À Eletronorte pelo apoio ao desenvolvimento do projeto "Uso de Varistores com Tecnologia Nacional à Base de $\mathrm{SnO}_{2}$ em Pára-raios com Substituição aos de ZnO hoje utilizados”, Ciclo 2004/2005 P\&D-Contrato 4500058143.

\section{REFERÊNCIAS}

[1] T. K. Gupta, A. C. Miler, J. Mater. Res. 3, 4 (1988) 745. [2] T. Miyosh, K. Maede, K. Takahashi, T. Yamazaki, Advances in Ceramics. Am. Ceram. Soc., Columbus, OH, EUA (1981) 309.

[3] S. R. Dhage, V. Ravi, S. K. Date, Bull. Mater. Sci. 27, 1 (2004) 43.

[4] S. A. Pianaro, P. R. Bueno, P. Olivi, E. Longo, J. A. Varela, J. Mater. Sci. Lett. 16, 8 (1997) 634.

[5] G.-Z. Zang, J.-F. Wang, H.-C. Chen, W.-B. Su, C.-M. Wang, P. Qi, J. Phys. D: Appl. Phys. 38 (2005) 1072.

[6] F. M. Verbi, F. Angel, I. M. G. Santos, J. A. Cerri, E. R. Leite, E. Longo, J. A. Varela, Anais $42^{\circ}$ Cong. Bras. Cerâmica, Poços de Caldas, MG, 2 (1998) 472.

[7] E. C. F. Souza, S. R. M. Antunes, S. A. Pianaro, A. J. Zara, A. C. Antunes, Anais XIII CBECIMAT, Curitiba, PR (1998) 2437.

[8] P. A. Santos, S. Maruchin, G. F. Menegoto, A. J. Zara, S. A. Pianaro, Mater. Lett. 60, 12 (2006) 1554.

[9] W.-X. Wang, J.-F. Wang, H.-C. Chen, W.-B. Su, G.-Z. Zang, Mater. Sci. Eng. B. 99 (2003) 470.

[10] P. R. Bueno, M. R. de Cassia-Santos, E. R. Leite, E. Longo, J. Bisquert, G. Garcia-Belmonte, F. FabregatSantiago, J. Appl. Phys. 88, 11 (2000) 6545.

[11] P. R. Bueno, E. R. Leite, M. M. Oliveira, M. O. Orlandi, E. Longo, Appl. Phys. Lett. 79, 1 (2001) 48.

[12] P. R. Bueno, M. M. Oliveira, W. K. Bacelar-Jr., E. R. Leite, E. Longo, G. Garcia-Belmonte, J. Bisquert, J. Appl. Phys. 91, 9 (2002) 6007.

[13] M. R. Cassia-Santos, P. R. Bueno, E. Longo, J. A. Varela, J. Eur. Ceram. Soc. 21, 2 (2001) 161.

[14] W. K. Bacelar, P. R. Bueno, E. R. Leite, E. Longo, J. A. Varela, J. Eur. Ceram. Soc. 26 (2006) 1221.

[15] J.-F. Wang, H.-C. Chen, W.-B. Su, G.-Z. Zang, C.-J. Zhang, C.-M. Wang, P. Qi, J. Electroceram. 14, 2 (2005) 133.

[16] C.-M. Wang, J.-F. Wang, W.-B. Su, H.-C. Chen, C.L. Wang, J.-L. Zhang, G.-Z. Zang, P. Qi, Z.-G. Gai, B.-Q. Ming, Mater. Sci. Eng. B. 127 (2006) 112.

[17] C.-M. Wang, J.-F. Wang, H.-C. Chen, W.-B. Su, G.-Z. 
Zang, P. Qi , Chinese Phys. Lett. 21, 4 (2004) 716.

[18] P. Qi, J.-F. Wang, W.-B. Su, H.-C. Chen, G.-Z. Zang, C.-M. Wang, B.-Q. Ming, Mater. Chem. Phys. 92, 2 (2005) 578.

[19] R. Parra, M. S. Castro, J. A. Varela, J. Eur. Ceram. Soc. 25 (2005) 401.

[20] R. Parra, Y. Maniette, J. A. Varela, M. S. Castro, Mater. Chem. Phys. 94, 2 (2005) 347.

[21] A. C. Antunes, L. A. Pinheiro, S. R. M. Antunes, E. Longo, J. A. Varela, Anais $41^{\circ}$ Cong. Bras. Cerâmica, S. Paulo, SP 1 (1997) 82.

[22] M. M. Oliveira, P. C. Soares, P. R. Bueno, E. R. Leite, E. Longo, J. A. Varela, J. Eur. Ceram. Soc. 23, 11 (2003) 1875.

[23] C.-M. Wang, J.-F. Wang, H.-C. Chen, W.-B. Su, G.-Z. Zang, P. Qi, Mater. Chem. Phys. 92, 1 (2005) 118.

[24] C. W. Nahm, Mater. Lett. 59 (2005) 2097.

[25] G. Z. Zang, J. F. Wang, H. C. Chen, W. B. Su, W. X. Wang, C. M. Wang, P. Qi, J. Non-Cryst. Solids 351 (2005) 941.

[26] J.-F. Wang, W.-B. Su, H.-C. Chen, W.-X. Wang, G.-Z. Zang, C.-P. Li, S. Bodde, J. Am. Ceram. Soc. 88, 2 (2005) 331.

[27] C.-M. Wang, J.-F. Wang, H.-C. Chen, W.-B. Su, G.Z. Zang, P. Qi, B.-Q. Ming, Solid State Comm. 132 (2004) 163.

[28] W.-X. Wang, J.-F. Wang, H.-C. Chen, W. Su, B. Jiang, G.-Z. Zang, C.-M. Wang, P. Qi, Ceram. Int. 31, 2 (2005) 287.

[29] G. Zang, J. Wang, H. Chen, W. Su, W. Wang, C. Wang, P. Qi, J. Alloy. Compd. 377 (2004) 82.

[30] G. Z. Zang, Physica B. 367 (2005) 29.
[31] P. Qi, J. F. Wang, W. B. Su, H. C. Chen, G. Z. Zang, C. M. Wang, B. Q Ming, Mater. Sci. Eng. B. 119 (2005) 94. [32] F. M. Filho, A. Z. Simões, A. Ries, L. Perazolli, E. Longo, J. A. Varela, Ceram. Int. 32 (2006) 283.

[33] P. R. Bueno, S. A. Pianaro, E. C. Pereira, L. O. S. Bulhões, E. Longo, J. A. Varela, J. Appl Phys. 84, 7 (1998) 3700 .

[34] M. M. Oliveira, Tese de Doutorado, Universidade Federal de S. Carlos, S. Carlos, S.P. (2002).

[35] P. R. Bueno, M. R. Cássia-Santos, E. R. Leite, E. Longo, J. Bisquert, G. Garcia-Belmonte, F. Fabregat-Santiago. J. Appl. Phys. 88, 11 (2000) 6545.

[36] A. Iga, M. Matsouka, T. I. Masuyama, Jap. J. Appl. Phys. 15, 6 (1976) 1161.

[37] A. Iga, M. Matsouka, , T. I. Masuyama, Jap. J. Appl. Phys. 15, 9 (1976) 1847.

[38] K. Eda, J. Appl. Phys. 49, 5 (1978) 2067.

[39] E. Olson, G. L. Dunlop, J. Appl. Phys. 66, 8 (1989) 3666 .

[40] C. -Y. Shen, Y. -C. Chen, L. Wu, Jap. J. Appl. Phys. 32, 5 (1993) 1147.

[41] G. E. Pike, C. H. Seager, Bull. Am. Phys. Soc. 23, 3 (1978) 291.

[42] G. Blatter, F. Greuter, Phys. Rev. B: Condens. Matter. 33, 6 (1986) 3952.

[43] S. Fujitsu, H. Toyoda, H. Yanagida, J. Am. Ceram. Soc. 70, 4 (1987) C-71.

[44] S. Fujitsu, H. Toyoda, H. Yanagida, Y. Watanabe, H. Kawazoe, Jap. J. Appl Phys. 38, 3 (1999) 1534.

[45] T. K. Gupta, J. Am. Ceram. Soc. 73, 7 (1990) 1817.

(Rec. 05/09/2007, Rev. 24/09/2007, 14/12/2007, Ac. 01/02/2008.) 